\title{
Topical issue on cold quantum matter
}

The rapidly growing area of ultracold quantum gases has steadily broadened its scope since the first observation of Bose-Einstein condensation in 1995, and has matured into an independent field of physics at the crossroads between the traditional areas of Atomic, Molecular and Optical Physics, Quantum Physics, Condensed Matter Physics, Statistical Physics, and Chemical Physics. In the last few years, we have witnessed the demonstration of Fermi degeneracy in ultracold atomic gases, the identification of magnetic Feshbach resonances as a means to tune interatomic interactions, the observation of the Mott-insulator transition of atoms in a optical lattice, and the formation of ultracold molecules in a single internal (vibrational, rotational and hyperfine) quantum state, just to name some of the ground-breaking results. All these milestones resulted from a close interplay between experimental and theoretical efforts, which has been a key factor for the rapid progress in this interdisciplinary field.

The success of this field is based on three unique features of atomic and molecular systems at temperatures close to absolute zero: first, the entropy of the atomic and molecular gases can be reduced to a minimum by the use of advanced cooling and trapping methods on the one hand, and by the application of quantum-state engineering allowing one to prepare single motional and internal quantum states with high fidelity on the other hand. Second, the nature and the strength of the interaction between the particles in the ultracold gas can be tuned over a large range, e.g. by using Feshbach resonances. Thus, one can explore the transition from a non-interacting gas to a complex, strongly correlated system in a controlled way. Third, the geometry and the dimensionality of the system under investigation can be adjusted almost at will, rendering it possible, e.g., to investigate one-dimensional physics of interacting particles or to simulate correlations in solid-state systems by confining atoms and molecules in lattice structures.

Motivated by the great opportunities of the field, the European Science Foundation (ESF) coordinated a three-year EUROCORES Programme on "Cold Quantum Matter" under the acronym EuroQUAM, assembling many European leading groups in the field. Funding of the selected projects was provided by the participating national agencies. The program, which started in 2007, has provided an excellent platform for new joint endeavours and has fostered many active collaborations within Europe. Many of these new projects have just started to blossom, and it is now hoped that they can continue to flourish beyond the three-year limit of the EUROCORES scheme. The open and enthusiastic spirit of the participating groups in the network was reflected in the excellent presentations and vivid discussions at the final international conference of EuroQUAM on "Cold Quantum Matter: Achievements and Prospects", which was held in Ischgl in September 2010.

The selection of topics for this Topical Issue is oriented along the six Collaborative Research Projects within EuroQUAM, with each of the guest editors being the coordinator of one of these projects: Quantum Simulation using Cold Atoms in Optical Lattices; Fermionic Mixtures of Ultracold Atoms; Controlled Interactions in Quantum Gases of Metastable Atoms; Cavity-Mediated Molecular Cooling; Quantum-degenerate dipolar gases of bialkali molecules; and, Collisions of Cold Polar Molecules. Despite this link to EuroQUAM, the Call for Papers to this Topical Issue was open to the general scientific community without restrictions, following the general policy of EPJD concerning importance and novelty of the articles. Numerous articles were received and accepted from non-members of EuroQUAM. Therefore, this Topical Issue provides a good overview of the current status of the field from a European perspective.

This issue is organized according to the current trends in the field of Ultracold Quantum Matter. In the first part, state-of-the-art aspects of Bose-Einstein condensation of atomic gases are covered. Lercher et al. and Jenkin et al. describe efficient ways to create single and double species Bose condensates. Deuar et al. explore the role of scattered atoms in collisions of two Bose condensates. Theoretical aspects of scaling of the interaction in BECs are the topic of the article by Sarjonen, Saarela and Mazzanti. Kónya, Szirmai and Domokos investigate quantum phase transitions of a Bose condensate in an optical resonator. Bose gases in 1D and the breakdown of integrability in these systems are studied by Mazets. Surprising effects in the collision of solitons in a multi-component Bose condensate are theoretically found by Trippenbach.

The second part is devoted to degenerate Fermi gases and, in particular, mixtures of ultracold Fermions. Naik et al. determine and analyse Feshbach resonances in a Fermi-Fermi mixture of Li and Levinson and Petrov develop a diagrammatic approach to describe atom-dimer and dimer-dimer scattering in fermionic mixtures close to a Feshbach resonance. Massignan and Bruun theoretically find interesting signatures for repulsive polarons and itinerant ferromagnetism in strongly polarized Fermi gases. Collisions of two one-dimensional clouds of fermionic atoms with opposite spin polarizations in an optical lattice form the topic of the article of Kajala, Massel, and Törmä. 
Cold and ultracold molecules and their interactions are the subject of the third part. Deiglmayr et al. explore the population redistribution of rovibrational levels in optically trapped polar molecules, in particular the LiCs molecule. Gerdes et al. measure the Stark effect in the NaK molecule with relevance for ultracold molecule experiments. Lepers and Dulieu discuss interesting aspects of cold atom-molecule photoassociation. Cho et al. present recent progress towards the formation of ultracold RbCs molecules. In a theoretical article, Zinner and Bruun predict the emergence of density waves in a layered system of fermionic polar molecules. Tokunaga et al. discuss the prospects of sympathetic cooling of molecules in various types of traps, while Wallis et al. theoretically explore possible routes for the sympathetic cooling of NH molecules with ultracold Li atoms. Riedel et al. report on the accumulation of ground-state NH molecules in a static magnetic trap. Caruso et al. analyse the energy redistribution in quenching collisions of cesium dimers with He atoms. Janssen, van der Avoird, and Groenenboom discuss the role of magnetic dipolar interactions in cold and ultracold collisions. Scharfenberg et al. present an experimental and theoretical study on inelastic scattering of Stark decelerated $\mathrm{OH}$ radicals with rare-gas atoms.

The fourth part of this Topical Issue contains three papers on ultracold atoms in reduced geometries. Plötz and Wimberger show that ultracold atoms in an optical lattice manifest a realization of Landau-Zener-Stückelberg interferometry. Daley, Ye and Zoller explore opportunities of state-dependent lattices for quantum computing with alkaline-earth-metal atoms. Matveeva, Recati and Stringari study the effects of long-range dipolar interactions on a bilayer harmonically trapped gas.

The advancement of the field of cold quantum matter is intimately linked to the development and extension of concepts and methods for cooling and trapping of atoms and molecules, which form the fifth and final part of the Topical Issue. Ridinger et al. present a large atom number dual species magneto-optical trap for a mixture of fermionic Li and $\mathrm{K}$ atoms. Vexiau et al. explore optimal optical trapping strategies for ultracold $\mathrm{Cs}_{2}$ molecules. Villwock, Siol and Walther present first results on the magneto-optical trapping of mercury, while Feldker et al. report on the magneto-optical trapping of bosonic and fermionic Ne isotopes and their mixtures. Trimeche et al. propose a new approach to the magnetic deceleration of a supersonic beam in a traveling magnetic wave. Xuereb et al. compare efficiencies of optical cooling schemes relying upon coupling to an optical cavity. Safonov, Safonova and Yasnikov consider theoretically a scheme of double-resonance spectroscopy of a quantum gas exploiting interatomic interactions. Finally, Welzel et al. discuss the experimental feasibility of quantum simulation of coupled spin systems employing trapped ion crystals.

In our view, this eclectic, yet comprehensive collection of papers offers an excellent overview of the achievements and prospects of the field of ultracold quantum matter. In the reviewing process care was taken that related work was cited adequately. Therefore, many of the articles in this Topical Issue provide a good introduction into the relevant literature. We sincerely hope that our excitement about the research presented here is shared by the readers and that they will find this Topical Issue a useful reference for their own research.

We gratefully acknowledge the support of the European Science Foundation through the EUROCORES Programme "Cold Quantum Matter (EuroQUAM)", the national funding agencies for funding this Programme, and, in particular, the tireless efforts of Dr Ana Helman and Dr Farzam Ranjbaran from the ESF office. Special thanks go to the Ms Solange Guéhot and the editorial staff at EPJD for their excellent assistance with this Topical Issue.

Gerhard Birkl

University of Darmstadt, Germany

Christopher Foot

University of Oxford, UK

Tim Freegarde

University of Southampton, UK

Rudolf Grimm

University of Innsbruck, Austria

Jeremy M. Hutson

University of Durham, UK

Matthias Weidemüller

University of Heidelberg, Germany 